\title{
3dRNA v2.0: An Updated Web Server for RNA 3D Structure Prediction
}

\author{
Jun Wang ${ }^{\mathbb{D}}$, Jian Wang ${ }^{\mathbb{D}}$, Yanzhao Huang and Yi Xiao * \\ School of Physics and Key Laboratory of Molecular Biophysics of the Ministry of Education, \\ Huazhong University of Science and Technology, Wuhan 430074, China \\ * Correspondence: yxiao@hust.edu.cn; Tel.: +86-27-8755-6652; Fax: +86-27-8754-2219
}

Received: 9 May 2019; Accepted: 15 August 2019; Published: 23 August 2019

\begin{abstract}
D structures of RNAs are the basis for understanding their biological functions. However, experimentally solved RNA 3D structures are very limited in comparison with known RNA sequences up to now. Therefore, many computational methods have been proposed to solve this problem, including our 3dRNA. In recent years, 3dRNA has been greatly improved by adding several important features, including structure sampling, structure ranking and structure optimization under residue-residue restraints. Particularly, the optimization procedure with restraints enables 3dRNA to treat pseudoknots in a new way. These new features of 3dRNA can greatly promote its performance and have been integrated into the 3dRNA v2.0 web server. Here we introduce these new features in the 3dRNA v2.0 web server for the users.
\end{abstract}

Keywords: RNA structure prediction; scoring function; structure optimization

\section{Introduction}

Noncoding RNAs can catalyze and regulate many biochemical reactions in organisms [1]. Just like proteins, to understand these functions, it is important to know their 3D structures. However, the number of solved RNA 3D structures is still quite small compared with the large amount of non-coding RNA sequences and this becomes an obstacle to explore their functions. Therefore, many computational methods have been proposed for predicting the 3D structure of non-coding RNA, e.g., FARNA [2]/FARFAR [3], MC-Sym [4], NAST [5], iFoldRNA [6,7], ASSEMBLE [8], Vfold [9,10], RNAComposer [11,12], 3dRNA [13-15] and F-RAG [16].

3dRNA is an automated method of building RNA 3D structures from sequences and secondary structures by using the smallest secondary elements (SSEs) [14]. The SSEs are defined as stem (helix), hairpin loop, internal loop, bulge loop, pseudoknot loop and junction loop. 3dRNA has been used by many researchers in practice [16-23]. Up to now, it has performed over 5500 tasks from other groups and was visited by more than 6000 individual IP addresses. The 3dRNA v2.0 web server can be accessed at http://biophy.hust.edu.cn/3dRNA.

\section{Results}

Our original 3dRNA web server can only do one type of task: users just need to input the sequence and secondary structure of an RNA and click the "submit" button, after which they can obtain its predicted structures automatically $[14,15]$. Recently, we have extended 3dRNA to include a method to further optimize the predicted 3D structures using the restraints obtained from experiments or theoretical predictions [24]. This optimization method can greatly increase the accuracy of the predicted structures using 3dRNA, as well as other RNA 3D structure prediction methods [24]. Furthermore, we have also implemented an algorithm of direct coupling analysis (DCA) [25] to find the most likely contacted nucleotide pairs that can be used as the restraints in the optimization procedure. In particular, 
the optimization method enables 3dRNA to treat pseudoknots in a new way. Instead of finding 3D templates for pseudoknots, 3dRNA takes the base pairs in the pseudoknots as restraints and then optimizes the predicted initial RNA 3D structures with the restraints (see the right bottom of Figure 1). It avoids the problem of missing templates for pseudoknots. On the other hand, since sampling and optimization processes usually are time-consuming, it is better to let users determine whether they need these processes or not. We have greatly modified the 3dRNA web server to incorporate these new features into it, which are colored red in Figure 1, and make it easy to use.

\section{1. $3 d R N A$}

Here we briefly describe the main steps of 3dRNA. Detailed descriptions of them can be found in our previous papers [13-15,24], and they can be elucidated by the schema diagram in Figure 1. Firstly, we decompose an RNA secondary structure into a tree, each node of which corresponds to an SSE and each edge connects two successive SSEs. Secondly, we search the template library and obtain a suitable 3D template for each of the nodes. Thirdly, we assemble the selected template of each node with that of its parent node. The Kabsch method [26] is used as the superposition method. After traversing through the tree, we can get a complete tertiary structure. For those nodes (SSE) that have more than one 3D template, the template having the highest sequence homology with the query SSE will be selected firstly. If users want to get more than one prediction, other templates will be selected randomly. In the case of missing 3D templates for a specific SSE, 3dRNA will use a bi-residues method (see Section 4 ) or distance-geometry method $[27,28]$ to generate its 3D templates. As an option, users of the 3dRNA web server can generate 3D templates for all SSEs of an RNA using these two methods and use them to assemble the RNA structure instead of the 3D templates extracted from the experimental structures. In the final step, 3dRNA clusters the candidates using the k-means algorithm and then uses the 3dRNAscore [13] to evaluate the centroids of each cluster. Then, users are free to choose the appropriate structures from them or feed them to the optimization procedure with addition of restraints from experiments and/or theoretical predictions [24]. It is noted that 3dRNA has no size limitation, though the accuracy will become lower for longer junctions.

The SSE 3D templates library has been updated. It is divided into two major sub-libraries: helix library and loop library. The helix library includes all kinds of helices extracted from experimentally determined RNA structures of different lengths and different sequences. Usually, there is more than one template for an SSE. The loop library consists of different types of loops including hairpin loops, bulge loops, internal loops and multi-branch loops. Two base pairs are attached to the ends of every loop, thus we can assemble two successive templates more accurately by overlapping them. Hairpin loops, bulge loops and internal loops involve all available types and sequences. The multi-branch loops (or named junction) contain 3-way junction, 4-way junction, 5-way junction, and so on. Currently, there are 27,163 helices and 27,826 loops in the library.

It has been shown that the prediction accuracy of 3dRNA and other widely used RNA 3D structure prediction methods could be improved significantly by the restraints from coevolutionary information obtained using direct coupling analysis (DCA) [24]. For instance, the averaged prediction accuracy is $9.61 \AA$ and $8.05 \AA$ for a small test set which contains 5 short RNAs (78 nt in average, PDB (Protein Data Bank) ID: 1FIR [29], 1Y26 [30], 2GDI [31], 3Q3Z [32], 4LVV [33]), with or without the DCA restraints, respectively. Particularly, nearly all these RNAs (except 2GDI) have pseudoknot, which is always a problem for RNA 3D structure predictions.

The schema diagram of the optimization procedure is shown in Figure 2 and the details can be found in our previous paper [24]. Given the initial RNA structure, 3dRNA will set the movable elements of it according to the secondary structure, which may be given by the user or calculated from the initial structure. The movable elements are all possible fragments that do not interrupt any helices, which keeps the secondary structure unchanged during optimization. In the simulated annealing Monte Carlo (SAMC) optimization process, a randomly chosen moveable element will be translated, rotated around a point, or rotated around an axis. If there are any restraints (DCA or distance), the 
optimization process can be done under them. Then, a set of conformations are sampled and clustered by using the k-means clustering algorithm according to their Root-Mean-Square-Deviation (RMSD) values from each other. The centroid of each cluster is determined and ranked by 3dRNAscore [13]. Finally, the ranked top $\mathrm{N}$ predictions are given to the user. It is worth noting that this clustering step is valuable as it can produce final predictions which are dissimilar to each other. All of these routines have been integrated into our new web server.

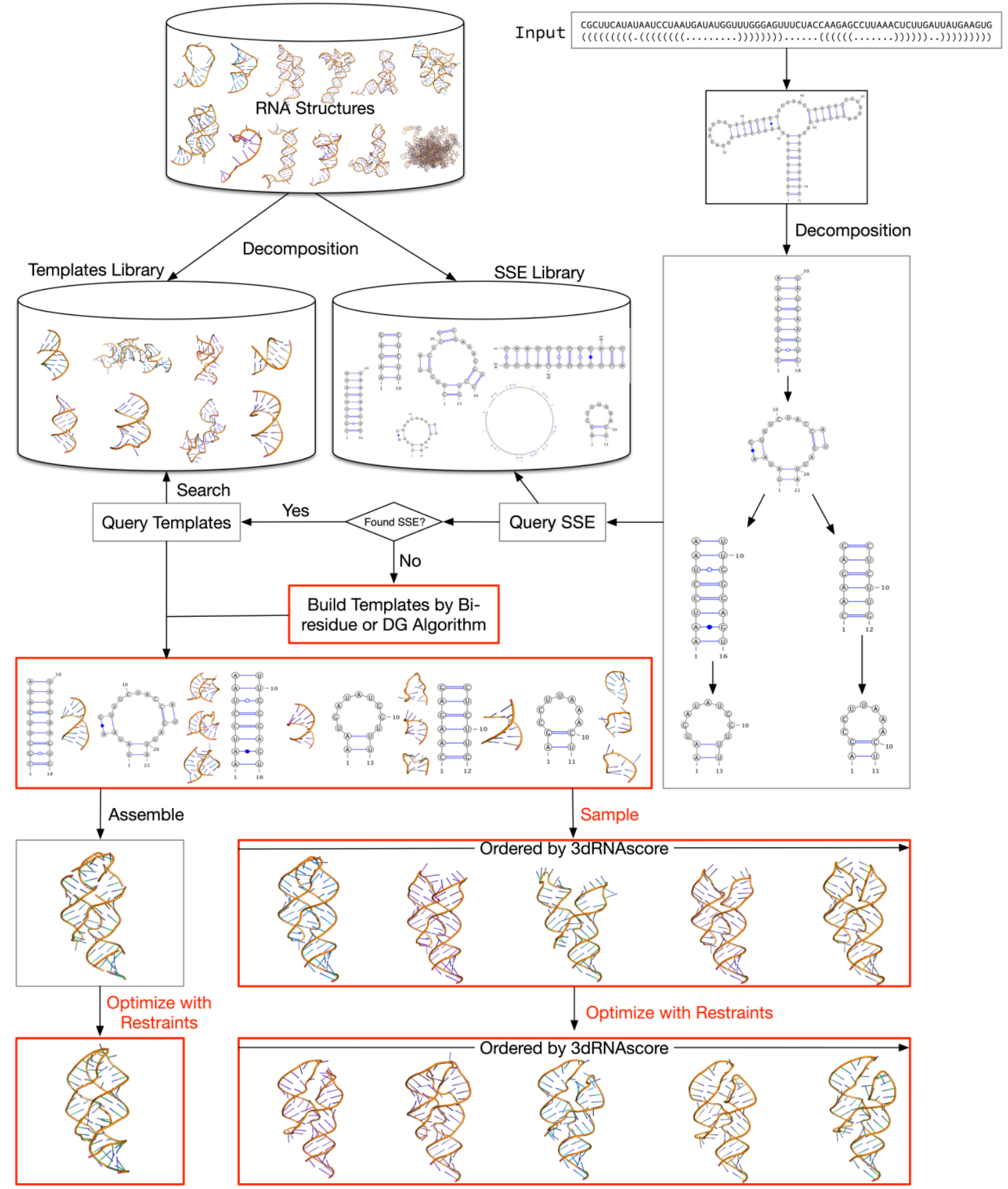

Figure 1. Schematic diagram of the prediction process of an RNA with pseudoknot (PDB ID: 1Y26 [30]) using 3dRNA v2.0. The red colored parts of this diagram are the new features compared with our previous web server. 


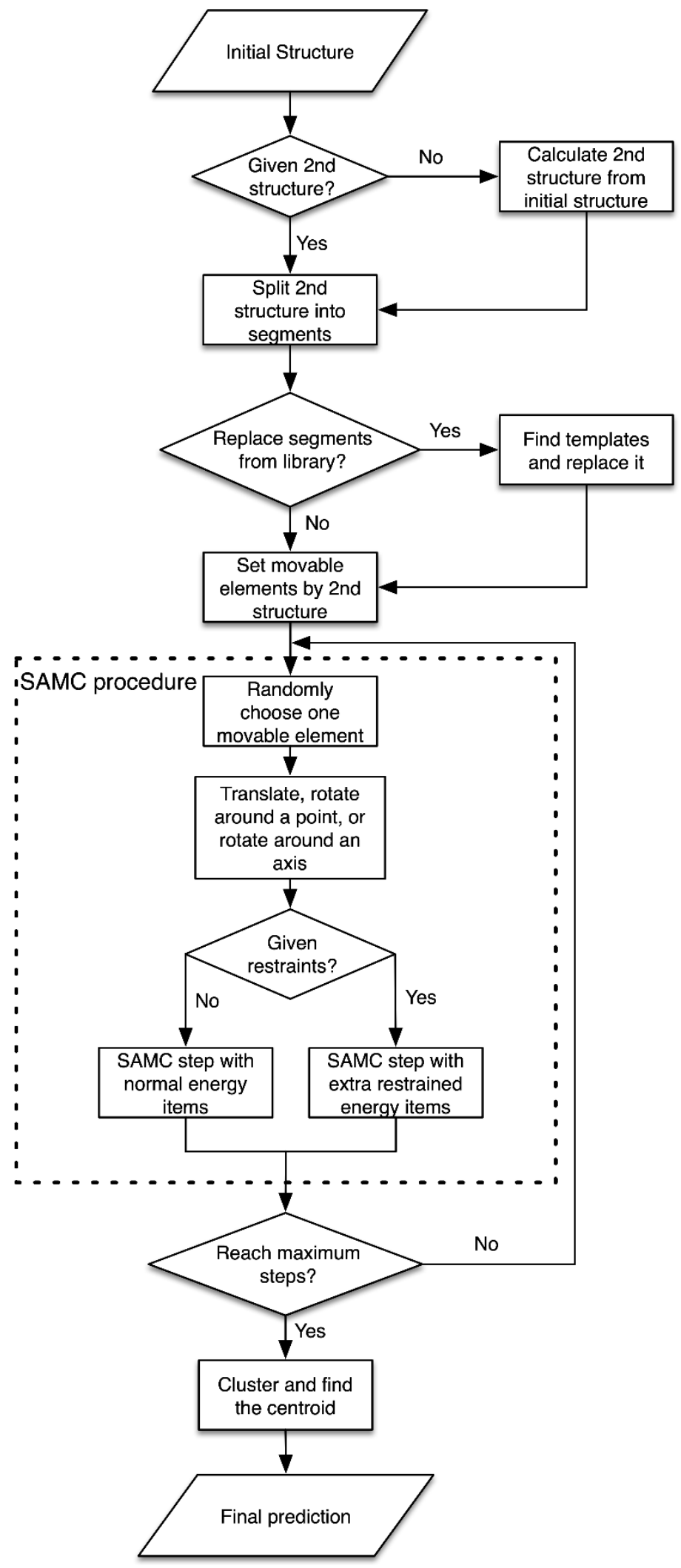

Figure 2. The flowchart of optimization procedure in 3dRNA. SAMC—simulated annealing Monte Carlo sampling method. 


\subsection{User Interface}

3dRNA web server has a main page that contains a new task area, a task query area, and an area for references. The UI of the new task area is shown in Figure 3. Most of the time, you will just need to input the sequence and secondary structure of an RNA and the type of task you are going to run, though several advanced options are available when you want to customize your prediction, such as the loop building method and the excluded PDB IDs in the circumstance that you want to validate and test our prediction method. Users can directly use 3dRNA without registering. The main change of the 3dRNA web server is in the new task area, where users are now free to choose different "Task type".

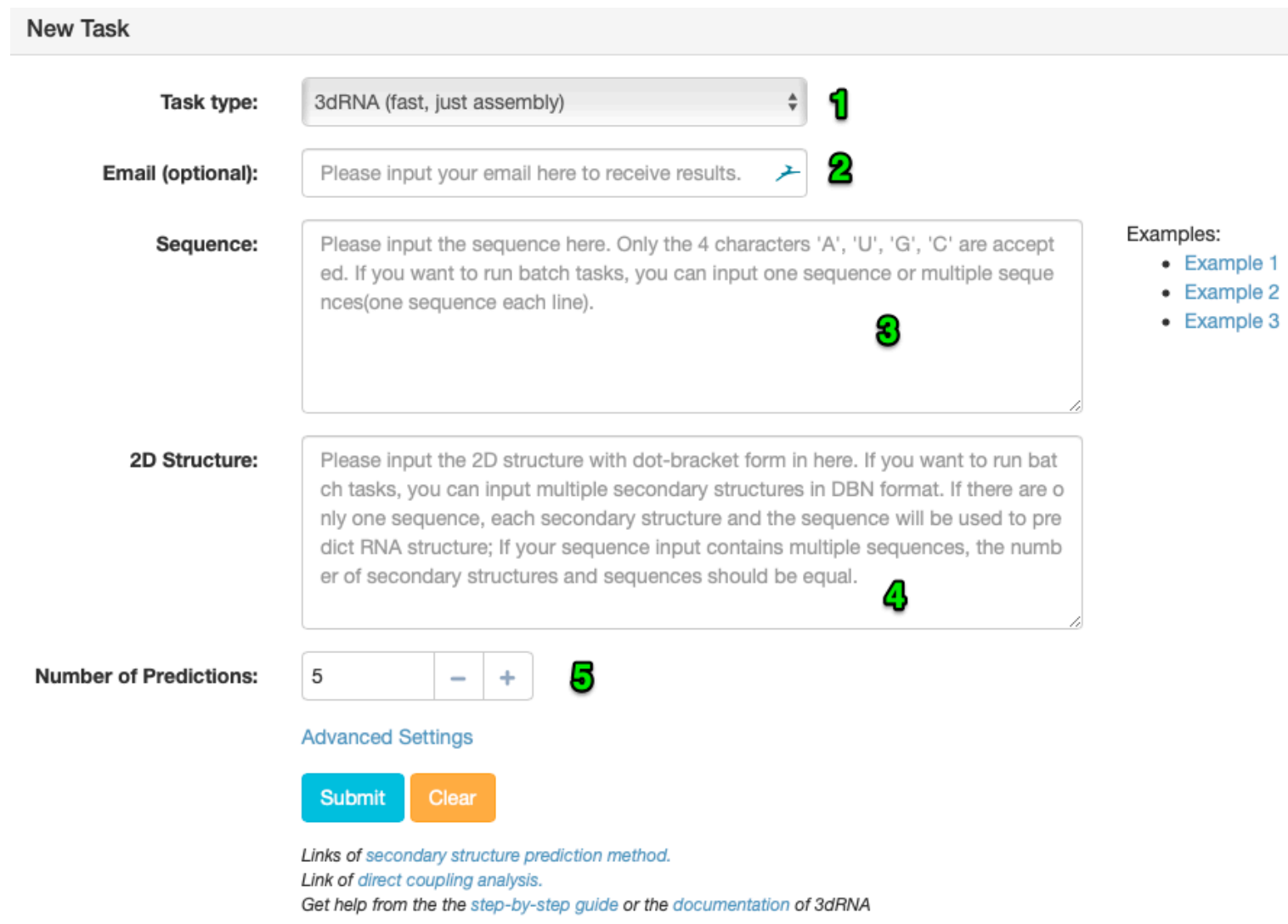

Figure 3. The main page of the 3dRNA web server. The fields labelled with a number in the figure are as follows: (1) "Task Type" field is used to select one of the task types in the following section. (2) The email you want to use to get notification after the job is finished; (3) A "Sequence" field is a required field, which is the sequence of the RNA you want to predict. (4) The "2D structure" field is the corresponding secondary structure for the sequence. (5) The "Number of Predictions" field is used to specify the number of final predictions you want to get.

There are four types of tasks:

1. 3dRNA (fast, just assembly): This is the most basic task of 3dRNA. It just finds 3D templates for each SSE and then assembles one or more sets of templates together as the final predictions. The number of assembled structures, $\mathrm{N}$, is determined by the number of predictions set by users (the default is 5). Among the $\mathrm{N}$ assembled structures, the first one is assembled using the top-ranked template of each SSE, which has the same secondary structure and most similar sequence with that of the query. The other N-1 assembled structures are assembled from randomly chosen templates of each SSE. The assembled structures will be refined through an energy minimization procedure using AMBER [34] in default. Therefore, this type of task is fast in speed.

2. 3dRNA with sampling (medium, assembly $\rightarrow$ sampling): For this type of task, 3dRNA firstly builds a structure by assembling the top-ranked template of each SSE and then samples different 
templates for each of them. At each sampling step, the 3D structure of each SSE is replaced by a randomly chosen candidate from available templates for the SSE in order to increase the diversity of predictions. Users are able to set the number of sampling steps (the default is 500). After that, the sampled structures are clustered by the analytical routine which uses a k-means algorithm in 3dRNA, and the centroids of all clusters are evaluated by our 3dRNAscore program. The number of clusters is determined by the "Number of Predictions" field set by users (the default is 5). Similarly, the finally chosen structures will be refined using the energy minimization procedure of AMBER by default. Obviously, this type of task is slower than the one above.

3. 3dRNA with optimization (slow, assembly $\rightarrow$ optimization): In this type of task, 3dRNA firstly assembles 3D structures in the same way as in task (1), and then it optimizes each of the assembled structures [24]. Users are capable of running the optimization procedure with or without restraints. There are two kinds of restraints acceptable: base pairs (like those given by DCA) or nucleotide-nucleotide distances. The DCA information is off-the-shelf from our DCA web server (http://biophy.hust.edu.cn/DCA). Inside our server, the inputted RNA pseudoknots in dot-bracket format are converted to restraints automatically. Therefore, to predict the 3D structure of an RNA with pseudoknots, you need to choose task type "3dRNA with optimization (slow, assembly $\rightarrow$ optimization)". The speed of the optimization procedure is much slower than that the above two types of tasks. It takes about $30 \mathrm{~min}$ to optimize a 75-nt-long RNA on our server, which has an Intel(R) Xeon(R) CPU E5620 @ 2.40 GHz CPU. However, our optimization method shows an approximately linear relationship between the running time and the sequence length. For example, for an RNA of about 400 nucleotides, the overall time of the optimization procedure is about $100 \mathrm{~min}$ [24].

4. Optimization: In this case, the optimization procedure is a standalone function for optimizing a given RNA structure. Users are capable of optimizing their uploaded RNA structures in PDB format, with or without the restraints described above.

\subsection{Cases}

3dRNA has a particular advantage in predicting long RNAs after the addition of the optimization procedure. Take the ribosomal RNA (PDB ID: 4ADV) with a length of 1410 nucleotides [35] as an example. The all-atom RMSD of the best predicted structure is $36.9 \AA$, and the Interaction Network Fidelity (INF) of canonical Watson-Crick base pairs and base stacking are 0.91 and 0.81 , respectively. Figure 4a shows the complicated 2D structure of 4ADV plotted by Forna [36], and Figure 4b shows the predicted and native 3D structure of it. The overall shape of the predicted structure is similar to the experimental one. Other examples can be found in ref [24]. 3dRNA v2.0 can predict 3D structures of RNAs with arbitrary size and any complicated topology, although the prediction accuracy needs further improvement.

As mentioned above, 3dRNA v2.0 treats pseudoknots using optimization under restraints. Figure 5 shows an example, the frameshift stimulating hairpin-type mRNA pseudoknot (PDBID: 2AP5, 28 nucleotides) [37]. The secondary structure of it is displayed in Figure 5a, and the predicted structures are shown in Figure $5 b-d$, respectively. For this RNA with pseudoknot interactions, our optimization without restraints can only slightly reduce the RMSD of the assembled structure relative to native structure from about $13.2 \AA$ to $12.2 \AA$. However, by taking pseudoknot as restraints, the optimization can decrease the RMSD value hugely from about $12.2 \AA$ to $5.9 \AA$, which can be seen clearly from Figure $5 c$,d. The INF of the canonical Watson-Crick base pairs and base stacking of the final predicted structure are 0.63 and 0.67 , respectively. 

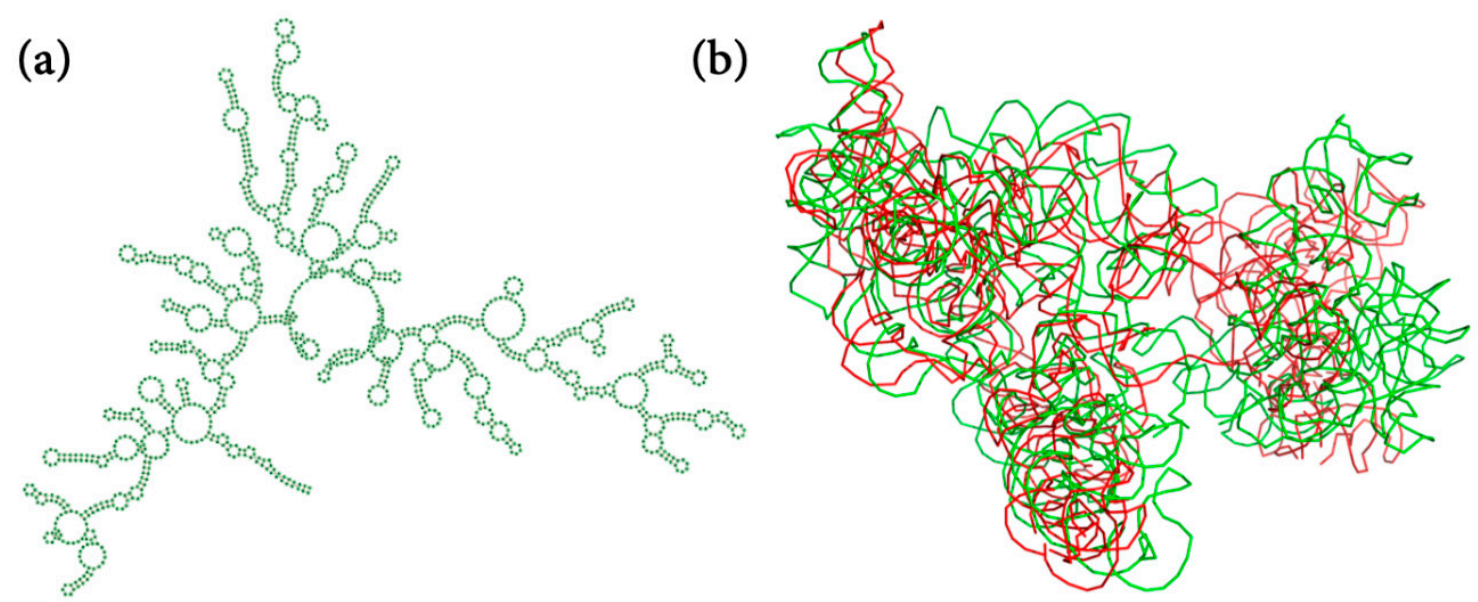

Figure 4. The secondary structure (a) and predicted 3D structure (b) of 4ADV (1410 nucleotides). The native and predicted structures are in green and red, respectively.

(a)

(c)

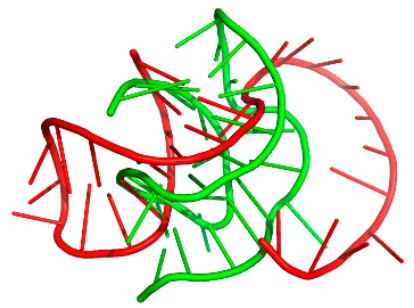

(b)

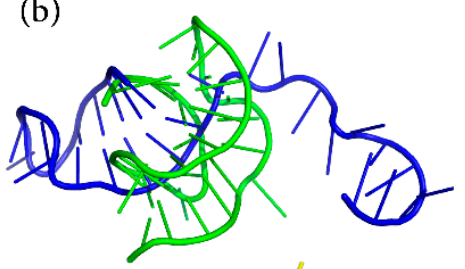

(d)

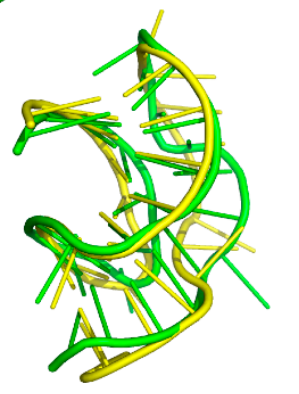

Figure 5. The secondary structure and predicted 3D structures for an RNA (PDB ID 2AP5): (a) is its secondary structure, (b) is the assembled structure (blue), (c,d) are the optimized structures not using (red) and using (yellow) the pseudoknot interactions as restraints, respectively. The native structure is represented in green cartoon.

\subsection{Server Architecture}

In order to facilitate the users to do a lot of predictions using our web server. Our web server can do RNA 3D structure predictions in batch mode now. Two modes are accepted-one sequence with different secondary structures and multiple sequences with the same number of secondary structures in DBN (dot bracket notation) format. In the former mode, you should input only one sequence in the field labelled number " 3 " in Figure 3 and multiple secondary structure strings (one for each line). The backend of our server will do predictions for each of these secondary structures. In the later mode, the RNA sequences and secondary structures in the field labelled number " 3 " and " 4 " should contain the same number of lines. Each line of sequence and the corresponding line of secondary structure will be used to do predictions. After the batch job is submitted successfully, the task IDs will be shown in a dialog. If you provide an email, the results will be sent to you after the job is finished.

The web server is based on Linux, Nginx, MySQL and PHP (LNMP) and built on a Laravel framework (https://laravel.com). The frontend is created using HTML5, CSS3, vue.js, JavaScript and Ajax, and the backend job manager is SLURM (https://www.schedmd.com/). The predicted structures are displayed using Jmol in the result page (http://www.jmol.org/). 


\section{Discussion}

The large RMSD of the predicted structures for large and complex RNA may stem from two reasons. The first one is the inaccuracy or lack of SSE templates, especially for multi-branch junctions; the second one is the absence of long-range tertiary interaction information. The former pitfall affects the spatial arrangement of SSE templates connected to the multi-branch junctions directly, and the latter one leads to the ignorance of some important restraints that may greatly improve the prediction accuracy. Besides this, the accuracy of RNA secondary structure prediction also significantly affects that of 3D structure prediction [38]. Therefore, in order to solve the problem, we may move along these directions in the future.

\section{Materials and Methods}

\subsection{Prediction Methods}

The predicted structures are built from the sequence and secondary structure, while the former is obtained from their native structures fetched from PDB (https://www.rcsb.org/), and the latter is calculated from DSSR (Dissecting the Spatial Structure of RNA) [39]. In the assembling process, which is able to assemble structures from sequence and secondary structure using templates from solved native structures, the templates that come from the same PDB ID are excluded to test the general performance of $3 \mathrm{dRNA}$. In order to get the multiple sequence alignment from the target sequence, the Infernal package [40] is used. The DCA results are calculated using our DCA web server (http://biophy.hust.edu.cn/DCA) and only preserve the top $\mathrm{N}$ values, while the $\mathrm{N}$ is calculated by multiplying the RNA sequence length and the factor 0.2. All initial structures that are the start points of optimization are attained by the assembling procedure in 3dRNA.

\subsection{Simulated Annealing Monte Carlo Simulation}

At each step of the optimization process, we choose one movable element randomly, which is determined by the secondary structure of RNA. Then, the translation procedure or rotation procedure is applied to the selected element. After that, the energy of the new conformation is calculated, and the Metropolis algorithm is used to determine whether this moving is accepted or rejected. However, sometimes the structures are trapped in local minima and the traditional Monte Carlo simulation is not enough to overcome the potential barrier. Thus, we use the simulated annealing Monte Carlo (SAMC) simulation method to optimize RNA structures. The simulated annealing protocol is composed of two steps: firstly, the simulated system is heated from low temperature to high temperature; secondly, the simulated system is cooled from high temperature to low temperature. This two-step process is easy to implement than the traditional Monte Carlo simulation procedure and usually improves the sampling efficiency.

\subsection{The k-Means Clustering Method}

After the optimization procedure, there will be a large amount of conformations. In order to get more than one representative structure from this ensemble, we use the k-means clustering method. The number of final predictions you want to get is the number of clusters (say the k super parameter in $\mathrm{k}$-means). The distance for a pair of conformations is the RMSD between them. In a k-means clustering algorithm, there is a method to determine the mean point for each cluster. In our program, the mean point in each cluster is the one with the smallest sum of distance to all other points, which can be represented by the formula below:

$$
\mathrm{s}_{\mathrm{i}}=\underset{\mathrm{k}}{\operatorname{argmin}} \sum_{\mathrm{j}, \mathrm{j} \neq \mathrm{i}}^{\mathrm{c}_{\mathrm{i}}} \mathrm{d}_{\mathrm{k}, \mathrm{j}}
$$

where $c_{i}$ is the number of points in ith cluster, $j, k$ is the index of points, and $d_{k, j}$ is the distance between jth and kth point. $s_{i}$ is the centroid of the ith cluster. 


\subsection{Bi-Residue Method}

The bi-residue method is used to generate 3D loop motifs of arbitrary types and it works as follows: (i) break the $5 \mathrm{~S}$ ribosome RNA structure into segments of two continuous nucleotides (bi-residue templates); (ii) build an initial chain of the SSE by randomly choosing templates from these segments; (iii) replace the bi-residues in the chain in random position by randomly choosing a bi-residue template; (iv) accept or reject the replacement using the Metropolis algorithm, and after a number of steps, the template of the SSE is generated.

\subsection{Distance Geometry}

The distance-geometry (DG) method is used to generate 3D loop motifs of arbitrary types and it works as follows: we first get statistics of distances between atoms within a nucleotide, between adjacent nucleotides, between paired nucleotides and between stacked nucleotides, respectively, from the known RNA 3D structures in the PDB. From these statistics we can determine the upper and the lower limits of these distances. Then, we can generate the distances between atoms of the residues for a given loop, which are between the corresponding upper and lower limits. Finally, the 3D coordinates can be obtained using the EMBED algorithm utilized in DG $[27,28]$.

\section{Conclusions}

We have added new features in our 3dRNA web server v2.0, including the sampling procedure and optimization procedure. All users are free to submit their tasks according to their needs. The input of our web server is easy to get and understand, and the running time for different tasks is reasonable. Moreover, the predicted results are reliable, and the UI is user friendly.

Author Contributions: Conceptualization, Y.X.; methodology, Y.X., J.W. (Jun Wang) and J.W. (Jian Wang); software, J.W. (Jun Wang) and J.W. (Jian Wang); validation, J.W. (Jun Wang) and Y.H.; formal analysis, J.W. (Jun Wang) and Y.H.; writing—original draft preparation, J.W. (Jun Wang) and J.W. (Jian Wang); writing-review and editing, Y.X.; visualization, J.W. (Jun Wang); supervision, Y.X.; funding acquisition, Y.X.

Funding: This work is supported by the NSFC under Grant No. 31570722 and 11874162.

Acknowledgments: We thank Shengyou Huang for valuable discussion and good advice about the web server.

Conflicts of Interest: The authors declare no conflicts of interest.

\section{References}

1. Mandal, M.; Breaker, R.R. Gene regulation by riboswitches. Nat. Rev. Mol. Cell. Biol. 2004, 5, 451-463. [CrossRef] [PubMed]

2. Das, R.; Baker, D. Automated de novo prediction of native-like RNA tertiary structures. Proc. Natl. Acad. Sci. USA 2007, 104, 14664-14669. [CrossRef] [PubMed]

3. Das, R.; Karanicolas, J.; Baker, D. Atomic accuracy in predicting and designing noncanonical RNA structure. Nat. Methods 2010, 7, 291-294. [CrossRef] [PubMed]

4. Parisien, M.; Major, F. The MC-Fold and MC-Sym pipeline infers RNA structure from sequence data. Nature 2008, 452, 51-55. [CrossRef] [PubMed]

5. Jonikas, M.A.; Radmer, R.J.; Laederach, A.; Das, R.; Pearlman, S.; Herschlag, D.; Altman, R.B. Coarse-grained modeling of large RNA molecules with knowledge-based potentials and structural filters. RNA 2009, 15, 189-199. [CrossRef] [PubMed]

6. Gherghe, C.M.; Leonard, C.W.; Ding, F.; Dokholyan, N.V.; Weeks, K.M. Native-like RNA tertiary structures using a sequence-encoded cleavage agent and refinement by discrete molecular dynamics. J. Am. Chem. Soc. 2009, 131, 2541-2546. [CrossRef] [PubMed]

7. Sharma, S.; Ding, F.; Dokholyan, N.V. iFoldRNA: Three-dimensional RNA structure prediction and folding. Bioinformatics 2008, 24, 1951-1952. [CrossRef]

8. Jossinet, F.; Ludwig, T.E.; Westhof, E. Assemble: An interactive graphical tool to analyze and build RNA architectures at the 2D and 3D levels. Bioinformatics 2010, 26, 2057-2059. [CrossRef] 
9. Cao, S.; Chen, S.J. Physics-based de novo prediction of RNA 3D structures. J. Phys. Chem. B 2011, 115, 4216-4226. [CrossRef]

10. Xu, X.; Zhao, P.; Chen, S.J. Vfold: A web server for RNA structure and folding thermodynamics prediction. PLOS ONE 2014, 9, e107504. [CrossRef]

11. Popenda, M.; Szachniuk, M.; Antczak, M.; Purzycka, K.J.; Lukasiak, P.; Bartol, N.; Blazewicz, J.; Adamiak, R.W. Automated 3D structure composition for large RNAs. Nucleic Acids Res. 2012, 40, e112. [CrossRef] [PubMed]

12. Biesiada, M.; Purzycka, K.J.; Szachniuk, M.; Blazewicz, J.; Adamiak, R.W. Automated RNA 3D Structure Prediction with RNAComposer. Methods Mol. Biol. 2016, 1490, 199-215. [CrossRef] [PubMed]

13. Wang, J.; Zhao, Y.; Zhu, C.; Xiao, Y. 3dRNAscore: A distance and torsion angle dependent evaluation function of 3D RNA structures. Nucleic Acids Res. 2015, 43, e63. [CrossRef] [PubMed]

14. Zhao, Y.; Huang, Y.; Gong, Z.; Wang, Y.; Man, J.; Xiao, Y. Automated and fast building of three-dimensional RNA structures. Sci. Rep. 2012, 2, 734. [CrossRef] [PubMed]

15. Wang, J.; Xiao, Y. Using 3dRNA for RNA 3-D Structure Prediction and Evaluation. Curr. Protoc. Bioinform. 2017, 57, 5-9. [CrossRef]

16. Jain, S.; Schlick, T. F-RAG: Generating Atomic Coordinates from RNA Graphs by Fragment Assembly. J. Mol. Biol. 2017, 429, 3587-3605. [CrossRef] [PubMed]

17. Cruz, J.W.; Sharp, J.D.; Hoffer, E.D.; Maehigashi, T.; Vvedenskaya, I.O.; Konkimalla, A.; Husson, R.N.; Nickels, B.E.; Dunham, C.M.; Woychik, N.A. Growth-regulating Mycobacterium tuberculosis VapC-mt4 toxin is an isoacceptor-specific tRNase. Nat. Commun. 2015, 6, 7480. [CrossRef]

18. Lu, T.; Ma, Q.; Yan, W.; Wang, Y.; Zhang, Y.; Zhao, L.; Chen, H. Selection of an aptamer against Muscovy duck parvovirus for highly sensitive rapid visual detection by label-free aptasensor. Talanta 2018, 176, 214-220. [CrossRef]

19. Rayaprolu, V.; Moore, A.; Wang, J.C.; Goh, B.C.; Perilla, J.R.; Zlotnick, A.; Mukhopadhyay, S. Length of encapsidated cargo impacts stability and structure of in vitro assembled alphavirus core-like particles. $J$. Phys. Condens. Matter. 2017, 29, 484003. [CrossRef]

20. Steuten, B.; Setny, P.; Zacharias, M.; Wagner, R. Mapping the spatial neighborhood of the regulatory 6S RNA bound to Escherichia coli RNA polymerase holoenzyme. J. Mol. Biol. 2013, 425, 3649-3661. [CrossRef]

21. Sun, M.; Li, W.; Blomqvist, K.; Das, S.; Hashem, Y.; Dvorin, J.D.; Frank, J. Dynamical features of the Plasmodium falciparum ribosome during translation. Nucleic Acids Res. 2015, 43, 10515-10524. [CrossRef] [PubMed]

22. Wang, X.; Vukovic, L.; Koh, H.R.; Schulten, K.; Myong, S. Dynamic profiling of double-stranded RNA binding proteins. Nucleic Acids Res. 2015, 43, 7566-7576. [CrossRef] [PubMed]

23. Jung, E.; Lee, J.; Hong, H.J.; Park, I.; Lee, Y. RNA recognition by a human antibody against brain cytoplasmic 200 RNA. RNA 2014, 20, 805-814. [CrossRef] [PubMed]

24. Wang, J.; Mao, K.; Zhao, Y.; Zeng, C.; Xiang, J.; Zhang, Y.; Xiao, Y. Optimization of RNA 3D structure prediction using evolutionary restraints of nucleotide-nucleotide interactions from direct coupling analysis. Nucleic Acids Res. 2017, 45, 6299-6309. [CrossRef] [PubMed]

25. Morcos, F.; Pagnani, A.; Lunt, B.; Bertolino, A.; Marks, D.S.; Sander, C.; Zecchina, R.; Onuchic, J.N.; Hwa, T.; Weigt, M. Direct-coupling analysis of residue coevolution captures native contacts across many protein families. Proc. Natl. Acad. Sci. USA 2011, 108, E1293-E1301. [CrossRef]

26. Kabsch, W. A discussion of the solution for the best rotation to relate two sets of vectors. Acta Crystallogr. Sect. A Cryst. Phys. Diffr. 1978, 34, 827-828. [CrossRef]

27. Havel, T.F. Distance geometry: Theory, algorithms, and chemical applications. Encycl. Comput. Chem. 1998, $120,723-742$.

28. Hubbard, J.M.; Hearst, J.E. Computer modeling 16 S ribosomal RNA. J. Mol. Biol. 1991, 221, 889-907. [CrossRef]

29. Benas, P.; Bec, G.; Keith, G.; Marquet, R.; Ehresmann, C.; Ehresmann, B.; Dumas, P. The crystal structure of HIV reverse-transcription primer tRNA(Lys,3) shows a canonical anticodon loop. RNA 2000, 6, 1347-1355. [CrossRef]

30. Serganov, A.; Yuan, Y.R.; Pikovskaya, O.; Polonskaia, A.; Malinina, L.; Phan, A.T.; Hobartner, C.; Micura, R.; Breaker, R.R.; Patel, D.J. Structural basis for discriminative regulation of gene expression by adenine- and guanine-sensing mRNAs. Chem. Biol. 2004, 11, 1729-1741. [CrossRef] 
31. Serganov, A.; Polonskaia, A.; Phan, A.T.; Breaker, R.R.; Patel, D.J. Structural basis for gene regulation by a thiamine pyrophosphate-sensing riboswitch. Nature 2006, 441, 1167-1171. [CrossRef] [PubMed]

32. Smith, K.D.; Shanahan, C.A.; Moore, E.L.; Simon, A.C.; Strobel, S.A. Structural basis of differential ligand recognition by two classes of bis- $\left(3^{\prime}-5^{\prime}\right)$-cyclic dimeric guanosine monophosphate-binding riboswitches. Proc. Natl. Acad. Sci. USA 2011, 108, 7757-7762. [CrossRef] [PubMed]

33. Trausch, J.J.; Batey, R.T. A disconnect between high-affinity binding and efficient regulation by antifolates and purines in the tetrahydrofolate riboswitch. Chem. Biol. 2014, 21, 205-216. [CrossRef] [PubMed]

34. Case, D.A.; Cheatham, T.E., 3rd; Darden, T.; Gohlke, H.; Luo, R.; Merz, K.M., Jr.; Onufriev, A.; Simmerling, C.; Wang, B.; Woods, R.J. The Amber biomolecular simulation programs. J. Comput. Chem. 2005, 26, 1668-1688. [CrossRef] [PubMed]

35. Boehringer, D.; O'Farrell, H.C.; Rife, J.P.; Ban, N. Structural insights into methyltransferase KsgA function in $30 \mathrm{~S}$ ribosomal subunit biogenesis. J. Biol. Chem. 2012, 287, 10453-10459. [CrossRef] [PubMed]

36. Kerpedjiev, P.; Hammer, S.; Hofacker, I.L. Forna (force-directed RNA): Simple and effective online RNA secondary structure diagrams. Bioinformatics 2015, 31, 3377-3379. [CrossRef] [PubMed]

37. Cornish, P.V.; Stammler, S.N.; Giedroc, D.P. The global structures of a wild-type and poorly functional plant luteoviral mRNA pseudoknot are essentially identical. RNA 2006, 12, 1959-1969. [CrossRef] [PubMed]

38. Zhao, Y.; Wang, J.; Zeng, C.; Xiao, Y. Evaluation of RNA secondary structure prediction for both base-pairing and topology. Biophys. Rep. 2018, 4, 123-132. [CrossRef]

39. Lu, X.J.; Bussemaker, H.J.; Olson, W.K. DSSR: An integrated software tool for dissecting the spatial structure of RNA. Nucleic Acids Res. 2015, 43, e142. [CrossRef]

40. Nawrocki, E.P.; Eddy, S.R. Infernal 1.1: 100-fold faster RNA homology searches. Bioinformatics 2013, 29, 2933-2935. [CrossRef]

(C) 2019 by the authors. Licensee MDPI, Basel, Switzerland. This article is an open access article distributed under the terms and conditions of the Creative Commons Attribution (CC BY) license (http://creativecommons.org/licenses/by/4.0/). 